\title{
Non-dipping morning blood pressure and isolated systolic hypertension in elderly
}

\author{
Zain-El Abdin MH, Snincak M, Pahuli K, Solarova Z, Hrabcakova P \\ Center for Research, Diagnosis and Treatment of Hypertension, Clinic of Geriatrics and Health Care, \\ School of Medicine, PJ Safarikiensis University, Kosice, Slovakia. mhzain@lf.upjs.sk
}

\begin{abstract}
Objective: Morning hypertension is currently the blind spot in the clinical practice of hypertension, home 24-hour blood pressure measurement has been recommended in patients with a high clinic blood pressure and patients with target organ damage.

Aim: To assess whether an increased early morning blood pressure surge, established via a single 24-hour blood pressure monitoring, in treated elderly hypertensive's is related to more prominent target organ damage. Material and methods: 310 treated hypertensive patients randomly attended the out-patient clinic of our hypertension centre. The patients were divided in 2 groups: systolic/diastolic blood pressure hypertension $(n=$ 266) and isolated systolic hypertension ( $n=4476 \pm 10 \mathrm{yrs}$ ). Results: Equivalent in both males and females, despite the rise in nondippers with an early morning surge (higher in females). Hyperlipidemia arises equally in dippers and nondippers, $45 \%$ of nondippers had diabetes mellitus; $30 \%$ suffered previous renal insufficiency and about $29 \%$ of patients suffered from an endocrine disorder, mostly thyroid. Metabolic syndrome was only found to be associated with a nondipping pattern (3\%). Left ventricular hypertrophy was present in $100 \%$ of patients, heart failure in $60 \%$ nondippers and $56 \%$ dippers, potential renal insufficiency in $34 \%$ nondippers and $22 \%$ dippers, retinopathy in $31 \%$ non dippers and $11 \%$ dippers, aortic aneurysm was only in nondippers. Conclusion: Early morning surge is a predictor of hypertensive target organ damage, being a dipper or nondipper patient with isolated systolic hypertension, might not make a difference depending on one's own degree of target organ damage and diurnal variation (Fig. 2, Ref. 30). Full Text in PDF www.elis.sk.

Key words: isolated systolic hypertension, early morning blood pressure surge, dippers, nondippers.
\end{abstract}

Despite a difficulty to diagnose and treat hypertension, this condition remains worldwide the leading cause of cardiovascular morbidity and mortality and goal blood pressure levels are seldom achieved. It is therefore highly desirable to improve this unsatisfactory delivery of care in the field of hypertension, an increasing number of clinical trials allow the formulation of guidelines to support a more effective strategy. Multifaceted interventions are required to implement guidelines successfully, going from the dissemination of recommendations to educational programs at the practice site. This paper is a current contribution to the above direction

The following are the main aims:

1) To prove the link between isolated systolic increase in blood pressure (non-dippers) and the incidence of hypertension in elderly subjects.

2) To compare the use of non- invasive blood pressure investigational methods versus management.

3) Prognosis of cardiovascular and cerebrovascular disease resulting from ISH in non-dippers?

Center for Research, Diagnosis and Treatment of Hypertension, Clinic of Geriatrics and Health Care, School of Medicine, PJ Safarikiensis University, Kosice, Slovakia

Address for correspondence: MH Zain Elabdin, MD, Center for Research, Diagnosis and Treatment of Hypertension, Clinic of Geriatrics and Health Care, School of Medicine, PJ Safarikiensis University, Trieda SNP 1, SK-040 01 Kosice, Slovakia

Phone: +421.902 .072009$

\section{Material and methods}

The extent to which the morning BP surge is a direct cause of cardiovascular disease is still to be proven, but a clear association has been demonstrated. An excessive morning blood pressure surge appears to be a risk factor for cardiovascular disease, particularly in older hypertensive patients with impaired auto regulation of hypertensive target organs $(1,2)$

There is no consensus on the definition of the morning BP surge. The classification of patients was made according to the Jichi Medical School ABPM Study (Wave1) (2) definition of morning blood pressure surge, the latter defined the 'sleep-trough surge' as the morning SBP minus the lowest SBP during sleep. The sleep -trough surge consists of two surges: the waking morning BP surge, defined as the morning SBP (the average of highest 4 morning values) minus the pre-awake SBP (average BP values between 4-6 am), and the prewaking morning BP surge (early morning surge EMS) is defined as the prewaking SBP minus the lowest SBP during sleep (between 12-4 am). As all patients were elderly, the exact time of waking wasn't available in all forms of patient's daily activity. Therefore, the surge used in this study was the prewaking morning BP surge (EMS).

However, it must be noted that other authors use other definitions: some authors define morning BP surge as morning SBP level measured on standing minus SBP before rising. This definition is relatively similar to that of waking surge. We defined morning BP 
surge as the average BP during morning period (4-6 am, average of 5 values) minus the average of the lowest three BP readings during the sleep period (12-4 am).

Our study, which lasted for a period of 2 and half years, included 310 elderly patients suffering from hypertension randomly, who visited the specialized center of treatment and diagnostics of hypertension in Kosice, Slovakia. The aim of the study was to investigate elderly patients with isolated systolic hypertension whom are 65 years of age and above. The ISH group $(n=44$ nondippers $\mathrm{n}=35$ and dippers $\mathrm{n}=9\}$ ) where divided according to age, sex, concomitant disease, medication, admission to hospital, visits to center, number of ABPM administration and hypertensive complications.

Variations were associated with an increased frequency of damage to all target organs (brain, heart and kidney) and a poorer prognosis for cardiovascular events was compared in patients with a normal dipping pattern versus nondippers. The frequency of this nondipping pattern increases with age, reaching approximately 50 $\%$ in elderly patients.

The early morning average BP from 4-6 am was calculated (the mean for all nondippers was $145.45 \mathrm{mmHg}$, SD $22.14 \mathrm{mmHg}$ ), the average BP of the lowest sleep values from 12-4 am (the mean of nondippers was $124.08 \mathrm{mmHg}$, SD $21.91 \mathrm{mmHg}$ ) and subtracted from the early morning average, the value obtained was the early morning blood pressure surge (EMBPS) or in other words the sleep- trough surge (mean $21.37 \mathrm{mmHg}$, SD $11.92 \mathrm{mmHg}, \mathrm{p}<$ 0.01 ). The mean sleep -trough surge is $37 \mathrm{mmHg}$ according to the Jichi Medical School ABPM Study (Wave1) (3). A cutoff value of $50 \mathrm{mmHg}$ was identified for the top decile (the surge group). The patient where sub classified $(37-50 \mathrm{mmHg}, \mathrm{n}=4$ : the morning surge group) versus all others ( $\mathrm{n}=31$ : the nonsurge group). Most patients had an EMBPS of $10 \mathrm{mmHg}$. However, some patients had higher sleep values than early morning values and therefore had no EMPS, although it might look different in the Holter graph it should be noted that only patients with BP values above the expected nocturnal BP were selected.

\section{Statistical analysis}

Values have been expressed as the means \pm SD or as percentages. Differences between groups were assessed by the analysis of variance (ANOVA) with the Mann-Whitney U test. The mean values for dipper and non-dipper patients were compared using the Student's t-test for independent samples. Chi-square statistics were used to compare categorical variables between groups.

\section{Results}

If a compelling disease exists, we noticed that antihypertensive medication had no independent role in controlling hypertension in nondippers; unless fully treated there will be always a dilemma in whether a specific antihypertensive medication could be effective enough in blood pressure reduction.

Most patients had an abnormal lipid profile and were on cholesterol lowering medication. Hyperlipidemia arises equally in the surge group and the nonsurge group. Almost half nondipping patients had DM; this further explains the $50 \%$ probability of the nondipping pattern arising from a compelling disease. Furthermore, $30 \%$ of patients with a nondipping pattern suffer renal insufficiency and about $29 \%$ of patients suffered from an endocrine disorder, mostly thyroid. Metabolic syndrome was only found to be associated with a nondipping pattern.

Nondippers with an early morning surge were more frequent in females. It is not definite weather females have a tendency to the nondipping pattern, it is thought that because the percentage of females suffering from isolated systolic hypertension in this study was higher than of males. Larger undergoing trails are still ongoing on the nocturnal pattern according to gender and medication.

In total $39 \%$ of patients, $14 \%$ dippers and $25 \%$ nondippers had a masked hypertension. Blood pressure was checked during clinic visit for ABPM administration and compared in the surge group (the mean $\mathrm{SBP}=146.66 \mathrm{mmHg}$, SD $26.45 \mathrm{mmHg}$, the mean $\mathrm{DBP}=82.22 \mathrm{mmHg}$, SD $15.63 \mathrm{mmHg}$ ) and the nonsurge group (the mean $\mathrm{SBP}=146.05 \mathrm{mmHg}$, SD $23.74 \mathrm{mmHg}$, the mean DBP $=82.71, \mathrm{SD} 14.13 \mathrm{mmHg}$ ).

The average pulse pressure in dippers was $64 \mathrm{mmHg}$ (SD 6.84 $\mathrm{mmHg}$ ) this high value plays a major role in target organ damage. In nondippers, it was $73.22 \mathrm{mmHg}$ (SD $13.94 \mathrm{mmHg}$ ), meaning an increase by about $10 \mathrm{mmHg}$ can be directly associated with the increase of cardiovascular and cerebrovascular incidents in elderly patients with ISH.

Fundoscopy, especially those who suffered from diabetes mellitus (it was found that $80 \%$ of patients who suffered from diabetes mellitus and isolated systolic hypertension had retinopathy).

All elderly patients with ISH had left ventricular hypertrophy. Heart failure was present in $90 \%$ of patients having EMS. MI and stroke were selected as independent ISH complications. Throughout the study, the prevalence of MI, stroke and other hypertensive complications was dependent on early morning surge, nondipping pattern and well controlled hypertension (dippers). Finally, aortic aneurysm was only noted from history.

The incidence of a MI and/or stroke was almost equal in (Dippers) and (ND without EMS), which in turn lead us to only differentiate between two groups (all pts versus nondippers with EMS). Females were the most affected group as they represent $82 \%$ of patients; they had the highest percentage of MI in a period of 2 and half years. Myocardial infarction (MI) in females and males was in a ratio of $4: 1$. With stroke, females also experienced cerebrovascular accidents more often than males, but not dipper males. There were no stroke cases in dipper males (Fig. 1).

\section{Discussion}

There are two relatively small prospective studies to support possible risk of morning BP surge. The first is the JMS ABPM study (Wave 1) carried out in elderly hypertensive patients (4) and the other is a French study of 519 hypertensive patients (the mean age 72 years) (4), where brain MRI was conducted to assess silent cerebrovascular disease together with 24-hr ABPM at baseline. The prognosis for stroke was studied during the follow up period of 41 months. Controlling for the cofactors and dipping status in 
Antihypertensive medication for morning hypertension

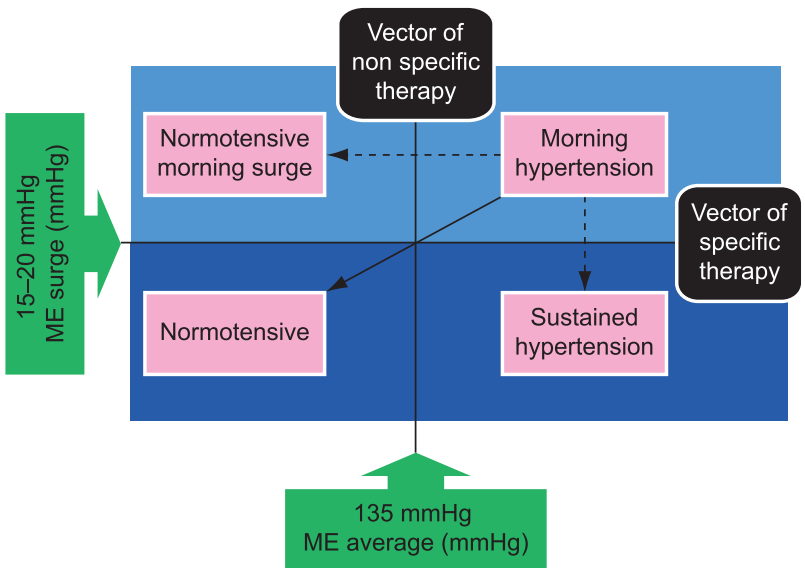

Fig. 1. Specific therapy - Long acting ARBs, ACE inhibitor, $\alpha$-adrenergic drugs with bedtime dosing, $\beta$ blockers, calcium channel blockers with negative chronotropic effects.

Nonspecific therapy - Long acting calcium channel blockers, diuretics. Adapted from Manual on Early Morning Risk Management in Hypertension (30).

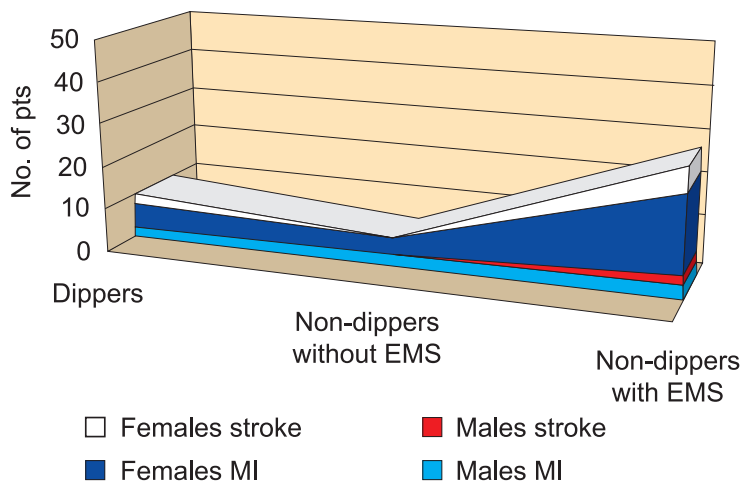

Fig. 2. Number of males and females with stroke and MI according to their dipping status.

the JMS ABPM study, sleep-trough surge was significantly and independently associated with stroke events and a morning surge of $10 \mathrm{mmHg}$ increased the clinical stroke risk by $25 \%$.

Another recent French prospective study in 507 patients with hypertension also found similar results (5).

In a cross-sectional study in newly diagnosed type 2 diabetic normotensive patients, morning BP levels and morning BP surge were significantly increased in patients with microalbuminuria compared to patients without microalbuminuria, despite this, there was no significant difference in the daytime BP and night-time BP between the two groups (5).

A few studies have reported a relationship between the ambulatory BP levels and LV hypertrophy (LVH) in ISH (5-7). However, one study (4) referred to the relationship between LVM and nocturnal BP decrease. In another report, there was not a significant difference in LVM between dipper and nondipper groups in ISH. This discrepancy was probably due to the difference in study subjects. In their study, normotensives were determined by rough criteria (clinical BP $<160 / 90 \mathrm{~mm} \mathrm{Hg}$, and awake $\mathrm{BP}<134 / 88 \mathrm{~mm}$ $\mathrm{Hg}$ ). Our subjects were selected by the criteria using the lower BP levels (clinic $\mathrm{BP}<140 / 90 \mathrm{~mm} \mathrm{Hg}$, and average 24-h ambulatory $\mathrm{BP}<125 / 80 \mathrm{~mm} \mathrm{Hg}$ ). Therefore, to clarify the association between the nondipping pattern and LVM, it might be necessary to investigate the subject group with lower BP levels. The effect of high BP might overwhelm the impact of nondipping on cardiac remodeling.

Patients with sleep disorders or breathing difficulties, such as sleep apnea, are likely to have a nondipping pattern of nocturnal BP. An increased frequency of micro arousals and related sympathetic activation, due to nocturnal hypoxia, may contribute to the nondipping pattern. Recent results showed that nocturnal physical activity was increased in nondippers compared to those with a normal nocturnal fall in BP. This increased physical activity maybe due, in part, to nocturnal behavior (going to bathroom, getting a drink of water, etc.) and poor sleep quality. It may also be possible that an increased frequency of unconscious micro arousals will trigger sympathetic activation.

The change of the sleep quality using hypnotics or antidepressants could affect nocturnal falls in BP and therefore, the development of silent and clinically overt cerebrovascular disease. Thus, an improvement in sleep quality together with a reduction of nocturnal physical activity may result in a more normal diurnal rhythm, especially in nondippers. This could also reduce the risk of cardiovascular disease in elderly patients (8).

In a recent prospective study on elderly patients, those with masked hypertension had the same cardiovascular risk as patients with sustained hypertension (9). In other recent studies in hypertensive patients taking medication, patients with masked hypertension had a significantly poorer cardiovascular prognosis than those with well controlled home (10) or ambulatory blood pressure levels (11) independent of clinic BP. The prevalence in the population of isolated office hypertension has been calculated and about 1 in 7 or 8 subjects with a normal office blood pressure may fall into this category (12). Although limited information exists on higher greater than normal prevalence of organ damage (13). Outcome studies have suggested that the masked hypertension increases cardiovascular risk, which appears to be close to that of in- and out-of-office hypertension $(14,15)$.

Several limitations of our study should be considered. The study population was small. However, carefully selecting and recruiting elderly individuals with recently diagnosed, well treated ISH is objectively difficult. Furthermore, some studies have found that day-night SBP differences in elderly persons (16) and classification of patients as dippers and nondippers are poorly reproducible over time $(17,18)$. On the other hand, the two study groups fulfilled all of the criteria normally used to define dipping and nondipping pattern.

The long follow-up of the PAMELA sample provides evidence that office, home, and ambulatory BPs are similarly predictive of the risk of cardiovascular and all-cause death, with superiority of systolic over diastolic BP and of night over daytime values. It further shows that the risk already increases at lower values and more steeply for ambulatory and home than for office BP. Finally, 
it shows that the different information provided by clinic and outof-clinic BP may be combined to improve prediction of risk (19).

\section{Conclusion}

Clinically relevant information can be utilized by simply having patients measure their blood pressure in the early morning, soon after arising (20), such readings are elevated in most patients. If they are elevated, the need for more effective, long-acting antihypertensive therapy is established. Moreover, the potential additional cardiovascular risk of heavy physical activity in the early morning can be avoided (21).

The mechanism, by which the morning BP surge might increase hypertensive target organ damage and trigger cardiovascular events in the morning hours, is unclear. In our study, the incidence of morning -onset stroke was significantly higher in the morning surge group than in the nonsurge group. Evidence is available that blood pressure normalization reduces the rate of cardiovascular events in hypertension and the prognostic value of $24 \mathrm{~h}$ ambulatory BP control being superior to that of office BP control. Our aim was to compare control of dipping status in patients with isolated systolic hypertension in office and check their morning BP values. The finding of good office BP control was able to reflect satisfactory control of ABP over 24 hours in a limited number of cases only. This emphasizes the need of a more frequent use of $24 \mathrm{~h} \mathrm{ABPM}$ in the management of hypertensive patients in general practice, in particular in patients with controlled office BP and a combination of 3 or more risk factors, such as, female gender, diabetes mellitus, advanced age and hyperlipidemia etc. An insufficient BP control by BP measurements performed in general practice requires confirmation by $24 \mathrm{~h}$ ABPM. The extent of target-organ damage has been positively related to the magnitude of blood pressure (BP) variability in hypertension. We evaluated the potential association between the 24-hour time rate of systolic blood pressure (SBP) variation derived from computerized analysis of ambulatory BP monitoring (ABPM) data and in complicated hypertensive target organ damage.

Masked hypertension (MH), dipping and the standard deviation (SD) of nighttime systolic blood pressure were the variability measures derived by a noninvasive ambulatory blood pressure monitoring (ABPM) in our study. The purpose of the present study was to identify predictors of progression and establishment of hypertensive complications during 2 and half-years in masked hypertensive's and sustained hypertension. After the diagnosis of masked hypertension, we conclude that risk factors contribute to the development of the observed increased morbidity in patients with masked hypertension, regarded as having an increased risk of cardiovascular morbidity and mortality, continuing monitoring of total cardiovascular risk profile is therefore important in these patients.

Pulse pressure (PP) is an established predictor of cardiovascular morbidity and mortality (23). PP was used in our study to evaluate the development in hypertensive complications in both dippers and nondippers. An increase in ambulatory PP was significantly higher in the group with nondipping pattern compared to the group with nonsurge pattern although both groups were treated for hypertension. Humans greatly vary in their circadian rhythms. These rhythms play a part in how a disease affects a person. Most of the nondipping patterns were associated with a compelling disease unlike dippers, but again it's uncertain that a compelling disease might be the cause of the nondipping pattern.

\section{News: Specific versus nonspecific antihypertensive treatment}

Figure 1 Shows the vector of specific antihypertensive medication (towards the baseline) and that of nonspecific medication (towards the left). Nonspecific medication for morning BP includes long acting calcium antagonists, such as amlodipine, and diuretics. The longer acting the antihypertensive, the better it is at controlling morning BP. These drugs are usually administered once daily in the morning and they provide continuous BP reduction over a $24 \mathrm{hr}$ period to attenuate exaggerated morning BP surge. In addition, more specific chronological treatment for morning BP surge may be achieved using an antihypertensive medication that reduces the presser effect of the nuerohumoral factors potentiated in the morning, such as inhibitors of the sympathetic activity or the RAAS.

Diuretics - diuretics provide a long duration of BP lowering effect and their effectiveness for the prevention of cardiovascular events is well established. However, when morning hypertension is treated using diuretics, nondippers shift towards becoming dippers, while the dipping pattern of dippers remains unchanged.

Calcium antagonists - some calcium antagonists have specific modes of action and could therefore provide more specific medication. The specific $\mathrm{T}$ - and $\mathrm{N}$-type calcium antagonist, who inhibits catecholamine release, is a possible candidate (24). Azelnidipine, another calcium antagonist, lowers the heart rate significantly when compared to amlodipine. In addition, pharmacokinetic ally, some extended-release forms of verapamil could also achieve effective $\mathrm{BP}$ reduction in the morning (25).

Alpha-adrenergic blockers and alpha/beta-adrenergic blockers - alpha-adrenergic and alpha/beta-adrenergic blockers are effective in reducing morning BP surge in hypertensive patients. In particular, night time dosing of alpha- adrenergic blockers achieves peak effect in the mornings, providing greater BP reductions during these hours.

There is no evidence that beta-adrenergic blockers reduce morning BP surge specifically, however, theoretically, there are effective for morning hypertensive patients with morning surge in heart rate. The effectiveness of beta-adrenergic blockers on cardio protection is well validated.

Angiotensin-converting enzyme inhibitors - ACE inhibitors such as ramipril used in the HOPE study, was found to have a better BP lowering effect during sleep than clinic and awake BP (26).

Angiotensin-receptor blockers- as well as ACE inhibitors, recent large clinical trials such as LIFE, SCOPE, and CHARM, have confirmed that ARBs are effective for target organ protection and for the prevention of cardiovascular events $(27,28)$.

Different ARBs have markedly different effects on morning BP levels and morning BP surge. Candesartan for example is su- 
perior to valsartan, as well as Diuretics, in decreasing morning BP and morning BP surge. Telmisartan is the ARB with the longest half-life (24-hours) and a meta-analysis of clinical efficacy has demonstrated superior BP reduction in morning hours.

Morning hypertension is more closely associated with diabetic complications than clinic hypertension. The lower target BP level of 130/80 for diabetic patients is now recommended for diabetic hypertensive patients. Strict control of morning hypertension, using ARBs that induce PPAR- $\gamma$ activity, is recommended for diabetic patients with metabolic syndrome.

\section{References}

1. Kario K, Shimada K, Pickering TG. Clinical implication of morning blood pressure surge in hypertension. J Cardiovasc Pharmacol 2003; 42 (Suppl): S87-S91.

2. Kario K, Pickering TG, Umeda Y et al. Morning surge in blood pressure as a predictor of silent and clinical cerebrovascular disease in elderly hypertensives: a prospective study. Circulation 2003; 107: 1401-1406.

3. Kario K, Schwartz JE Davidson KW et al. Gender differences in associations of diurnal blood pressure variation, awake physiacal activity and sleep quality with negative affect: The Work Site Blood Pressure Study. Hypertension 2001; 38: 997-1002.

4. Kario K, Pickering TG, Umeda Y et al. Morning surge in blood pressure as a predictor of silent and clinical cerebrovascular disease in elderly hypertensives: a prospective study. Circulation 2003; 107: 1401-1406.

5. Gosse $\mathbf{P}$, Lasserre R, Minifie $\mathbf{C}$ et al. Blood pressure surge on rising. J Hypertens 2004; 22: 1113-1118.

6. Caramori ML, Pecis M, Azevedo MJ. Increase in nocturnal blood pressure and progression to microalbuminuria in diabetes. N Engl J Med 2003; 348: 260-264.

7. Nakano S, Fukuda M, Hotta F et al. Reversed circadian blood pressure rhythm is associated with occurrences of both fatal and nonfatal vascular events in NIDDM subjects. Diabetes 1998; 47: 1501-1506.

8. Kapiotis S, Jilma B, Quehenberger $P$ et al. Morning hypercoagulability and hypofibrinolysis. Diurnal variations in circulating activated factor VII, prothrombin fragment F1+2, and plasmin-plasmin inhibitor complex. Circulation 1997; 96: 19-21.

9. Bjorklund K, Lind L, Zethelius B et al. Isolated ambulatory hypertension predicts cardiovascular morbidity in elderly men. Circulation 2003; 107: $1297-1302$.

10. Bobrie G, Chatellier G, Genes $\mathbf{N}$ et al. Cardiovascular prognosis of „masked hypertension“ detected by blood pressure self-measurement in elderly treated hypertensive patients. JAMA 2004; 291:1342-1349.

11. Clement DL, De Buyzere ML, De Bacquer DA et al. Office versus Ambulatory pressure Study investigators. Prognostic value of ambulatory blood-pressure recordings in patients with treated hypertension. $\mathrm{N}$ Engl J Med 2003; 348: 2407-215.

12. Shimada K, Kario K, Umeda Y et al. Early morning surge in blood pressure. Blood Press Monit 2001; 6: 349-353.

13. Kario K, Pickering TG. Blood pressure levels and risk of stroke in elderly patients. JAMA 2000; 284: 959-960.
14. Fagard RH, Van Den Broeke C, De Cort P. Prognostic significance of blood pressure measured in the office, at home and during ambulatory monitoring in older patients in general practice. J Hum Hypertens 2005; 19: 801-807.

15. Vaccarino $\mathbf{V}$ et al. Pulse pressure and risk of cardiac events in the Systolic Hypertenion in the Elderly (SHE) program. Amer J Cardiol 2001; 88 (9): 980-986.

16. James MA, Fotherby MD, Potter J F. Reproducibility of the circadian systolic blood pressure variation in the elderly. J Hypertens 1995; 13: 1097-1103.

17. Manning G, Rushton L, Donnelly R, Millar-Craig MW. Variability of diurnal changes in ambulatory blood pressure and nocturnal dipping status in untreated hypertensive and normotensive subjects. Am J Hypertens 2000; 13: 1035-1038.

18. Mochizuki J, Okutani M, Donfeng Y, Iwasaki H, Takusagawa M, Kohno I, Mochizuki S, Umetani K, Ishii H, Iyiri H, Komori S, Tamura K. Limited reproducibility of circadian variation in blood pressure dippers and nondippers. Am J Hypertens 1998; 11: 403-409.

19. Mancia G, Sega R, Grassi G, Cesana G, Zanchetti A. Defining ambulatory and home blood pressure normality: further considerations based on data from the PAMELA Study. J Hypertens 2001; 19: 995-999.

20. Gosse P, Cipriano C, Bemurat $\mathbf{L}$ et al. Prognostic significance of blood pressure measured on rising. J Human Hypertens 2001; 15: 413-417.

21. Leary AC, Struthers AD, Donnan PT, et al. The morning surge in blood pressure and heart rate is dependent on levels of physical activity after waking. J Hypertens 2002; 20: 865-870.

22. Carney RM, Freedland KE, Jaffe AS, Altered circadian pattern of acute myocardial infarction in patients with depression. Coron Artery Dis 1991; 2: 61-65.

23. Kario K, Mitsuhashi T, Shimada K. Neurohumoral characteristics of older hypertensive patients with abnormal nocturnal blood pressure dipping. Am J Hypertens 2002; 15: 531-537.

24. Kitahara Y, Saito F, Akao M et al. Effect of morning and bedtime dosing with cilnidipine on blood pressure, heart rate, and sympathetic nervous activity in essential hypertensive patients. J Cardiovasc Pharmacol 2004; 43: 68-73.

25. Wellington K, Scott LJ. Azelnidipine. Drugs 2003; 63: 2613-2621.

26. Svensson $P$, de Faire $\mathbf{U}$, Sleight $P$ et al. Comparative effects of ramipril on ambulatory and office blood pressures: a HOPE substudy. Hypertension 2001; 38: E28-E32.

27. Lithell H, Hansson L, Skoog I et al. SCOPE Study Group. The Study on Cognition and Prognosis in the Elderly (SCOPE): principal results of a randomized double-blind intervention trial. J Hypertens 2003; 21: 875-886.

28. Pfeffer MA, Swedberg K, Granger CB et al. CHARM Investigators and Committees. Effects of candesartan on mortality and morbidity in patients with chronic heart failure: the CHARM-Overall programme. Lancet 2003; 362: 759-766.

29. Kario K, Shimada K, Schwartz J, et al. Silent and clinically overt stroke in older Japanese subjects with white-coat and sustained hypertension. J Am Coll Cardiol 2001; 38: 238-245.

30. Kario K. Manual on Early Morning Risk Management in Hypertension. Clin Manual 2005: 41. 